\title{
Representações sociais da aprendizagem autodirigida entre médicos da atenção primária à saúde
}

\author{
Self-directed learning among primary health care doctors: an analysis in light of the theory of social representations \\ Gustavo Valadares Labanca Reis ${ }^{1}$ (1) gustavo.labanca@ufop.edu.br \\ Jucimere Fagundes Durães Rocha² (1) jucimerefdr@yahoo.com.br \\ Leonardo Cançado Monteiro Savassi' ${ }^{1}$ (D) savassi@ufop.edu.br \\ Cristina Andrade Sampaio 3 (D) cristina.sampaio@unimontes.br \\ Antônio Prates Caldeira ${ }^{3}$ (D) antonio.caldeira@unimontes.br
}

\section{RESUMO}

Introdução: Em um cenário de grande disponibilidade de informações, a produção de conhecimento científico em medicina tem sido cada dia mais acelerada. A forma como o profissional médico percebe e dirige sua aquisição de conhecimento ainda carece de estudos nacionais, particularmente em tempos de internet de fácil acesso.

Objetivo: Este estudo teve como objetivo analisar as representações sociais de médicos atuantes em equipes da atenção primária à saúde (APS) sobre aprendizagem autodirigida.

Método: Trata-se de um estudo quali-quantitativo fundamentado na Teoria das Representações Sociais de Moscovici com abordagem estrutural da Teoria do Núcleo Central de Abric, realizado em três municípios de Minas Gerais, no Brasil. Realizaram-se entrevistas semiestruturadas sobre o tema que foram gravadas e transcritas. As palavras evocadas livremente que surgiram do indutor "autoaprendizagem médica" foram analisadas com auxílio do software $\mathrm{EVOC}^{\circledR}$ por meio do quadro das quatro casas; e o software $\mathrm{CHIC}^{\circledR}$ analisou a similaridade. Para as falas dos participantes, realizou-se a análise de conteúdo.

Resultado: Efetuaram-se 50 entrevistas, e as palavras evocadas livremente, que possivelmente compõem o núcleo central das representações, foram "conhecimento", "dedicação", "estudo", “leitura”, "necessidade”, tendo como contraste "pesquisa” e "livro".

Conclusão: Os resultados demonstraram que as características do aprendiz e a prática como lócus de aprendizagem em contraposição à teoria, o qual está associado à barreira do tempo, definem o conteúdo central da representação social dos médicos participantes. Nesse contexto estudado, a APS reforça sua importância como cenário da autoaprendizagem médica.

Palavras-chave: Educação Médica; Educação Continuada; Aprendizagem; Atenção Primária à Saúde.

\section{ABSTRACT}

Introduction: In a scenario of a great information availability, the production of scientific knowledge in medicine has been increasingly accelerated. The way the medical professional perceives and directs their acquisition of knowledge still lacks national studies, particularly in times of easily accessible internet.

Objective: To analyze the social representations of physicians working in Primary Health Care (PHC) teams about self-directed learning.

Method: This is a qualitative-quantitative study based on Moscovici's Social Representations Theory, with a structural approach to Abric's Central Core Theory, conducted in three municipalities of the state of Minas Gerais, Brazil. Semi-structured interviews were conducted on the subject, which were recorded and transcribed. The freely evoked words that emerged from the inducing term "medical self-learning" were analyzed with the aid of EVOC" software through the four-quadrant chart and $\mathrm{CHIC}^{\circledast}$ software analyzed the similarity. Content analysis was performed for the participants' speeches.

Results: Fifty interviews were carried out and the freely evoked words that possibly constitute the core of the representations were "knowledge", "dedication", "study", "reading", "need", contrasting "research" and "book".

Conclusion: The results showed that the learners' characteristics, practice as a learning locus as opposed to theory, associated with the time barrier, define the core content of the social representation of the participating physicians. In this assessed context, PHC reinforces its importance as a scenario for medical self-learning.

Keywords: Medical Education; Continuing Education; Learning; Primary Health Care.

\footnotetext{
${ }^{1}$ Universidade Federal de Outro Preto, Outro Preto, Minas Gerais, Brasil.

${ }^{2}$ Faculdade de Saúde Ibituruna, Montes Claros, Minas Gerais, Brasil.

${ }^{3}$ Universidade Estadual de Montes Claros, Montes Claros, Minas Gerais, Brasil.
}

Editora-chefe: Rosiane Viana Zuza Diniz.

Editor associado: Roberto Zonato Esteves.

Recebido em 01/12/20; Aceito em 29/06/21.

Avaliado pelo processo de double blind review 


\section{INTRODUÇÃO}

A aprendizagem pode ser definida como o processo de aquisição de conhecimentos, habilidades e atitudes para uma mudança comportamental'. É um processo eminentemente pessoal, experiencial e que pressupõe uma mudança de comportamento ${ }^{2}$. As particularidades no processo de aprendizagem do adulto, em relação à criança, definiram uma nova área de estudos, a andragogia. Para os estudiosos da área, o adulto precisa entender o porquê da necessidade de aprender algo, trazendo consigo a influência de experiências anteriores. Aprendizes adultos estão orientados mais de forma temática, mais específica, com foco em tarefas e problemas reais, e possuem uma motivação interna para que possam melhorar ${ }^{2-4}$.

Knowles et al. $^{2}$ definem o aprendizado do adulto como autodirigido, ou seja, quando o indivíduo toma a iniciativa, com ajuda ou não de outrem, de avaliar as próprias necessidades de aprendizagem, definir as metas, implementar uma estratégia de aprendizado e avaliar os desfechos. Outro aspecto importante da aprendizagem do adulto é apontada por Kolb et al. ${ }^{5}$, que destacam o ciclo de aprendizagem experiencial (Figura 1 e Quadro 1). Para os autores, esse processo sofre influência da personalidade do aprendiz, das especificidades educacionais, da carreira profissional, do papel no seu trabalho e das competências adaptativas. Nesse sentido, o conhecimento é gerado pela transformação da experiência, em que primeiro existe uma vivência ou experiência concreta, que traz a observação e reflexão, seguida da conceitualização do que ocorre e de sua assimilação, e, assim, modifica sua atuação ${ }^{5}$.

Conhecer mais profundamente o processo de aprendizagem e construção do conhecimento é particularmente importante para o desenvolvimento de

Figura 1. Modelos explicativos de aprendizagem.

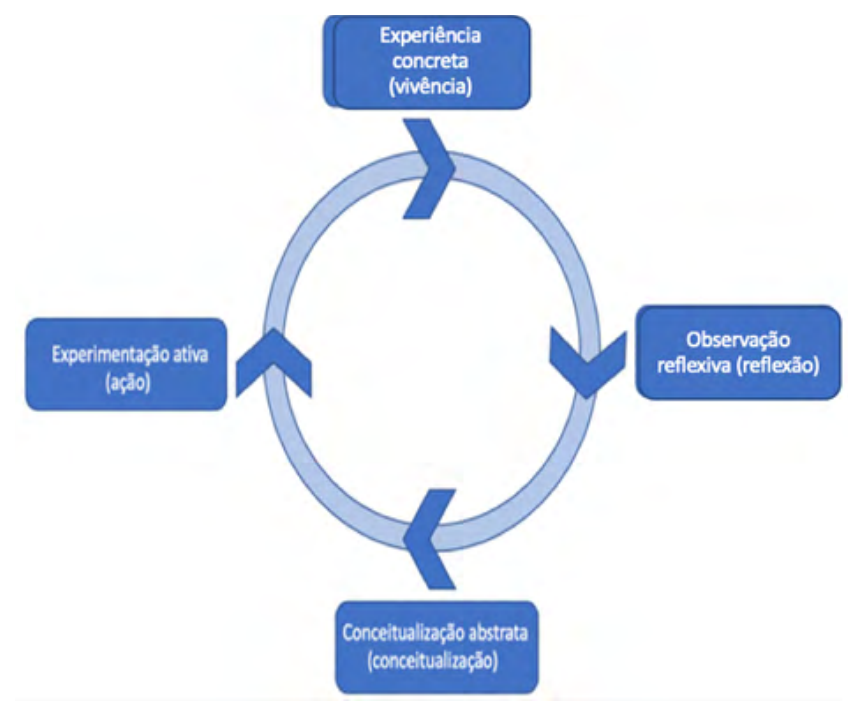

profissionais cujo processo de formação não se encerra com as atividades acadêmicas, como na medicina. $O$ médico vivencia, cotidianamente, oportunidades de aprendizagem. Na formação continuada desse profissional, a utilização de questões do cotidiano, aliada a experiências reais, tanto positivas quanto negativas, é considerada o ponto-chave no interesse dele em adquirir conhecimento ${ }^{6}$. Um estudo clássico de Slotnick, realizado no fim da década de 1990, traz uma análise da forma da aprendizagem autodirigida do médico que se baseia em quatro passos (Figura 1): identificação de uma necessidade de aprendizagem, busca de recursos para lidar com a necessidade de aprendizagem, aquisição de conhecimento e aplicação do conhecimento ou ganho de experiência ${ }^{7}$.

Entende-se que intervenções mais passivas, como aulas e congressos, tendem a ter pouca interferência no desempenho do médico e nos desfechos do cuidado do paciente. O tempo entre os esforços de educação continuada, como cursos e treinamentos, também parece ser inversamente correlacionado

Quadro 1. Comparativo de modelos explicativos de aprendizagem.

\begin{tabular}{ll}
\hline $\begin{array}{c}\text { Ciclo de aprendizagem } \\
\text { de Kolb et al. }{ }^{5}\end{array}$ & $\begin{array}{c}\text { Sequência de aprendizagem do } \\
\text { médico de Slotnick }\end{array}$ \\
\hline Experiência concreta & $\begin{array}{l}\text { Identificação de uma } \\
\text { necessidade de aprendizagem }\end{array}$ \\
Observação reflexiva & $\begin{array}{l}\text { Busca de recursos para lidar com } \\
\text { a necessidade de aprendizagem }\end{array}$ \\
Conceitualização abstrata & $\begin{array}{l}\text { Aquisição de conhecimento } \\
\text { Aplicação do conhecimento ou } \\
\text { ganho de experiência }\end{array}$ \\
\hline
\end{tabular}

Fonte: Kolb et al. ${ }^{5}$ e Slotnick ${ }^{7}$.

Fonte: Kolb et al. ${ }^{5}$ e Slotnick 7 .

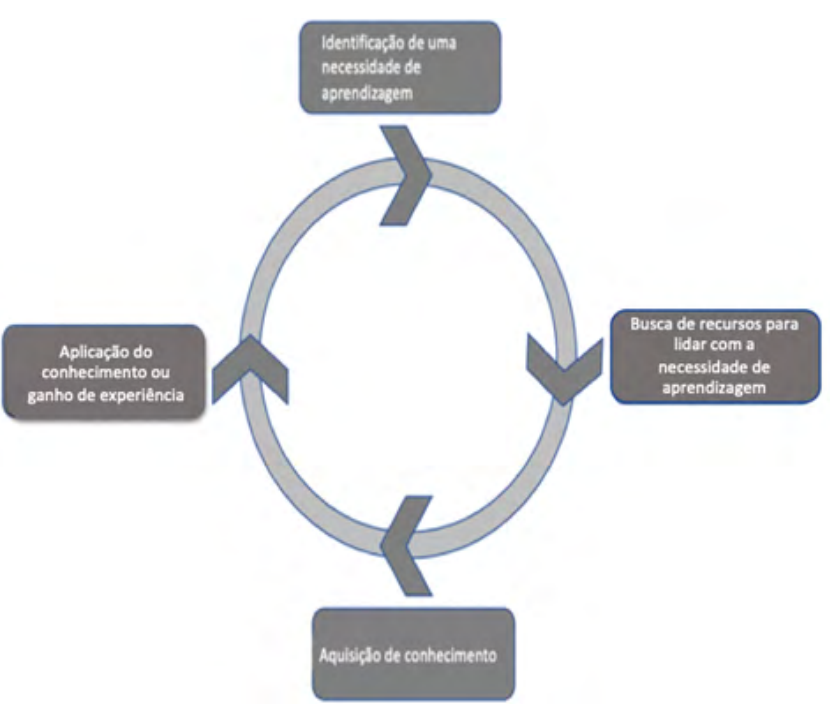


com o desempenho do médico. Em outras palavras, quanto mais tempo ele fica sem um novo treinamento, pior é o seu desempenho, reforçando o caráter contínuo e permanente da capacitação $0^{8,9}$. Denominam-se educação continuada, na prática médica, quaisquer meios pelos quais o médico aprende depois que completa seu treinamento formal, como forma de permitir mudanças na sua prática ${ }^{10,11}$.

Atualmente, a disponibilidade fácil às informações e as rápidas mudanças, aliadas ao uso de recursos tecnológicos para potencializar o aprendizado, destacam o protagonismo do aprendiz e remetem à aprendizagem autodeterminada, que reforça a inclusão de aspectos como a capacidade, criatividade e autoeficácia ${ }^{4}$. A difusão de dispositivos móveis trouxe uma nova realidade para os estudantes de Medicina e médicos ${ }^{12-14}$. O ensino a distância (EaD) com o uso de metodologias de aprendizagem baseada em problemas é capaz de possibilitar a aquisição de habilidades e conhecimentos em áreas remotas e atingir muitas pessoas, com desfechos semelhantes aos do modelo presencial ${ }^{15-17}$.

A atenção primária à saúde (APS) apresenta características distintas, pois amplia os aspectos para a formação de competências na graduação e pós-graduação médica. Nela, o aprendiz consegue vivenciar a relação do indivíduo, sua comunidade em um território, a ampliação do escopo da clínica e sua integração com outros saberes e profissionais, tendo o contato ao longo do tempo como aliado nesse processo ${ }^{18,19}$. Diferentemente do aspecto hospitalocêntrico, os aspectos culturais, o saber popular e a relação dos indivíduos com seu território são fatores importantes da APS $^{18}$.

O Brasil, desde a introdução das Diretrizes Curriculares Nacionais (DCN) de 2014, define a formação médica de caráter generalista, com competências distintas para as áreas da atenção à saúde, gestão em saúde e educação em saúde. Esse processo formativo privilegia o Sistema Único de Saúde (SUS) e, particularmente, a formação para atuação na APS. Contudo, tal orientação revelou carências no corpo docente das instituições formadoras, na infraestrutura de campos de prática e no modo de trabalho das equipes de saúde da $\mathrm{APS}^{20}$. A insuficiência da formação da graduação percebida na prática do trabalho na APS reforça a necessidade de aprimoramento e de oferta de pósgraduação ${ }^{21,22}$. Tais ações são capitaneadas por universidades federais, que ofertam gratuitamente processos de educação continuada a distância, nos formatos de aperfeiçoamento, capacitação ou especialização, proporcionando oportunidades de educação permanente, que podem se enquadrar nas necessidades de aprendizagem identificadas por profissionais de saúde. Para tanto, a estratégia do aprendizado é voltada para o saber-fazer, na qual objetivos de aprendizagem centram-se nas ações cotidianas e proporcionam aprendizado para a prática ${ }^{17,23,24}$.
Existem poucos estudos nacionais que avaliam o processo de aprendizagem entre médicos e sobre a percepção desses profissionais sobre o processo individual de aprendizagem. No contexto de fortalecimento e rápida expansão dos serviços de APS registrado no Brasil nos últimos anos, é pertinente conhecer as percepções dos médicos que atuam nesses serviços sobre autoaprendizagem. Essas informações podem facilitar o processo de educação permanente para esses profissionais, potencializando ações dos gestores de saúde e reduzindo custos. Nesse sentido, o objetivo do presente estudo foi analisar as representações sociais de médicos atuantes em equipes da APS sobre aprendizagem autodirigida.

\section{MÉTODOS}

Trata-se de um estudo exploratório, descritivo, quantiqualitativo, tendo como referencial teórico a Teoria das Representações Sociais ${ }^{25}$, por meio da abordagem estrutural da Teoria do Núcleo Central. Tal abordagem foi escolhida porque proporciona, por suas proposições, um refinamento conceitual e teórico-metodológico para o estudo das representações sociais. Conhecer as representações sociais torna possível desvendar o conhecimento comum de um grupo social. Esse conhecimento traz a real vivência e significância desse objeto, permitindo a compreensão do processo de aprendizagem em que esses indivíduos participantes se inserem ${ }^{26}$.

O estudo foi conduzido em Ouro Preto, Mariana e Viçosa, no estado de Minas Gerais. Não houve processo de amostragem. A coleta de dados realizou-se de forma censitária, considerando todos os 73 potenciais participantes, o que corresponde ao universo de médicos cadastrados e atuantes em equipes da Estratégia Saúde da Família (ESF) dos municípios. Os sujeitos envolvidos neste estudo são médicos atuantes há pelo menos seis meses e estão cadastrados em equipes da ESF dos municípios incluídos na pesquisa, tanto nas zonas rurais como nas urbanas. Foram excluídos docentes ou preceptores de estudantes de graduação para cursos na área da saúde, bem como aqueles que estavam afastados devido a férias, atestado médico ou licenças médicas.

A coleta de dados na pesquisa foi realizada por dois entrevistadores, por intermédio de um questionário produzido pelos pesquisadores, contendo questões fechadas e abertas. As questões fechadas buscaram caracterizar os entrevistados quanto aos aspectos sociodemográficos profissionais e de formação acadêmica. Já as questões abertas foram usadas para a técnica de evocação de palavras livres, por termo indutor: autoaprendizagem médica ${ }^{27}$. Solicitou-se a cada um dos participantes que produzisse cinco palavras, expressões ou adjetivos que viessem à mente, a partir da apresentação do tema indutor. Posteriormente, o participante deveria apontar qual a 
palavra ou expressão era a mais importante entre todas e, por último, justificar essa escolha. As três perguntas abertas foram gravadas e, posteriormente, transcritas para análise. Quando se analisam as palavras apresentadas espontaneamente, é possível colher elementos constitutivos do conteúdo de representação, de acordo com Abric ${ }^{28,29}$.

Os dados referentes ao perfil sociodemográfico e de formação acadêmica foram analisados por análise estatística descritiva simples com auxílio do IBM SPSS 23.0 para MAC. Os dados provenientes da técnica de evocação de palavras livres com o termo indutor autoaprendizagem médica foram analisados por meio do quadro de quatro casas de Pierre Vergès ${ }^{30}$, construído com o auxílio do software Ensemble de Programmes Permettant I'Analyse des Evocations (EVOC ${ }^{\circledR}$ ) versão $2005^{27}$.

Em seguida, realizou-se análise de similaridade por meio da árvore hierárquica de similaridade elaborada com auxílio do software Classification Hiérarchique Classificatoire et Cohésitive - CHIC $^{\circledR}$ (versão 4.1). Para essa análise, este estudo considerou todas as evocações presentes no quadro de quatro casas. Consideraram-se, por meio do software $\mathrm{CHIC}^{\circledR}$, as similaridades entre 0,5 e 1,00.

Finalmente, conduziu-se a análise dos registros gravados a partir das respostas às perguntas abertas sobre a principal palavra ou expressão da evocação. A técnica utilizada foi a análise de conteúdo ${ }^{31}$.

Todos os aspectos éticos foram respeitados para o desenvolvimento do presente estudo. O projeto foi aprovado por meio do Parecer n 3.040.247/2018 em Comitê de Ética em Pesquisa, devidamente registrado pela Comissão Nacional de Ética em Pesquisa (Conep). Os gestores das secretarias municipais de saúde dos municípios envolvidos no estudo e os médicos participantes assinaram o Termo de Consentimento Livre e Esclarecido. Asseguraram-se aos participantes o sigilo e a confidencialidade das informações.

\section{RESULTADOS}

Realizaram-se 50 entrevistas de dezembro de 2018 a fevereiro de 2019, nas três cidades propostas. Entre os 23 não entrevistados, registraram-se três negativas de participação e 20 pessoas que preenchiam critérios de exclusão, como afastamento por férias e trabalho como preceptor de alunos de graduação, principalmente.

Houve ligeiro predomínio do sexo feminino entre os participantes do estudo, com idade variando de 25 a 54 anos. $O$ tempo decorrido desde a formação em Medicina foi em média de 64,1 meses, sendo o tempo máximo de 300 e o mínimo de seis meses. Em termos de titulação, por volta de dois terços dos médicos eram generalistas (Tabela 1).
Dos participantes, metade relatou trabalhar em alguma atividade além da ESF, e $60 \%$ dos entrevistados faziam alguma atividade relacionada à aprendizagem. Dos que tinham atividades laborais além da ESF, quase $70 \%$ relataram atividades de atendimento a urgências em hospitais e unidades de pronto atendimento (UPA). Dos que relataram participar de atividades relacionadas à aprendizagem, cerca de um terço dos entrevistados fazia residência médica de medicina de família e comunidade, e a mesma proporção participava de atividades de EaD.

Em relação aos dados formados pelas evocações dos 50 médicos entrevistados, produziram-se 248 palavras ou expressões: 122 diferentes e, em média, 4,96 por participante. Foi considerada para essa construção a média das Ordens Média de Evocação (OME), ou seja, rang igual a 2,7, ao passo que a frequência média ficou estabelecida em 7 e a mínima em 4.

A análise dos resultados da evocação indica as tendências estruturantes (núcleo central e núcleo periférico) da representação social dos sujeitos entrevistados. Os

Tabela 1. Perfil dos médicos entrevistados - Ouro Preto, Viçosa e Mariana, Minas Gerais, 2018.

\begin{tabular}{|c|c|c|c|}
\hline Variável & & $\mathbf{N}$ & $\%$ \\
\hline \multirow[t]{3}{*}{ Gênero } & Feminino & 27 & 54,0 \\
\hline & Masculino & 23 & 46,0 \\
\hline & Total & 50 & 100,0 \\
\hline \multirow[t]{4}{*}{ Faixa etária } & De 20 a 30 anos & 26 & 52,0 \\
\hline & De 31 a 40 anos & 16 & 32,0 \\
\hline & Acima de 40 anos & 8 & 16,0 \\
\hline & Total & 50 & 100,0 \\
\hline \multirow[t]{5}{*}{ Tempo de formado } & Menos de 1 ano & 8 & 16,0 \\
\hline & De 1 a 5 anos & 26 & 52,0 \\
\hline & De 5 a 10 anos & 8 & 16,0 \\
\hline & Acima de 10 anos & 8 & 16,0 \\
\hline & Total & 50 & 100,0 \\
\hline \multirow{5}{*}{$\begin{array}{c}\text { Tempo de atuação na } \\
\text { ESF }\end{array}$} & Menos de 1 ano & 9 & 18,0 \\
\hline & De 1 a 5 anos & 29 & 58,0 \\
\hline & De 5 a 10 anos & 6 & 12,0 \\
\hline & Acima de 10 anos & 6 & 12,0 \\
\hline & Total & 50 & 100,0 \\
\hline \multirow[t]{4}{*}{ Cidade de atuação } & Ouro Preto & 19 & 38,0 \\
\hline & Viçosa & 17 & 34,0 \\
\hline & Mariana & 14 & 28,0 \\
\hline & Total & 50 & 100,0 \\
\hline \multirow[t]{4}{*}{ Formação } & Generalista & 29 & 58,0 \\
\hline & Especialista & 20 & 40,0 \\
\hline & Mestrado & 1 & 2,0 \\
\hline & Total & 50 & 100,0 \\
\hline
\end{tabular}


entrevistados associaram mais rápida e frequentemente a representação de autoaprendizagem às seguintes 16 evocações: conhecimento, dedicação, estudo, leitura, necessidade, internet, prática, tempo, artigos científicos, confiança, discussão de casos, experiência, interesse, melhoria, livro e pesquisa.

No quadrante superior esquerdo representado na Figura 2, que é composto pelos elementos considerados como provavelmente centrais, estão as palavras "conhecimento", "dedicação", "estudo", "leitura", "necessidade", que são os possíveis elementos centrais da representação social dos médicos estudados sobre autoaprendizagem médica.
A primeira e segunda periferias são formadas por nove evocações: "internet", "prática", "tempo", "artigos científicos", "confiança", "discussão de casos", "experiência", "interesse", "melhoria".

Posteriormente, as 16 evocações presentes no quadro de quatro casas foram submetidas à análise hierárquica de similaridade geradas a partir do software $\mathrm{CHIC}$, criando a árvore hierárquica de similaridade demonstrada na Figura 3.

Observa-se, na árvore de similaridade, que há três grandes grupos que não apresentam ligação entre si: um composto por "conhecimento", "confiança", "leitura" e "prática",

Figura 2. Quadro de quatro casas de distribuição da frequência e ordem média de posição gerada pelo Rangfrq do software EVOC ${ }^{\circledR}$ do banco de dados dos médicos entrevistados em 2018.

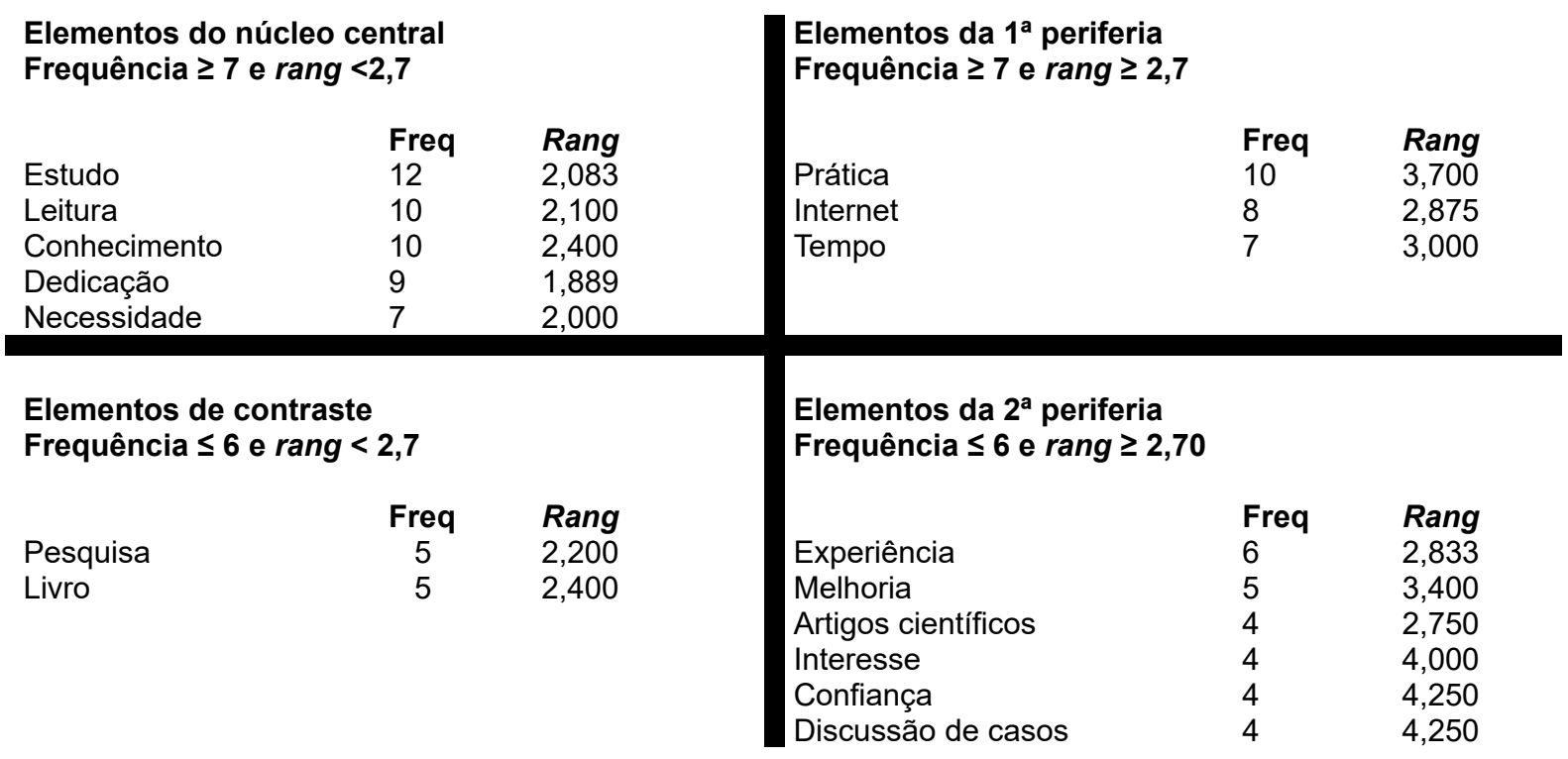

Figura 3. Árvore hierárquica de similaridade das categorias geradas pelo software $\mathrm{CHIC}^{\circledast}$ do banco de dados dos médicos pesquisados em 2018.

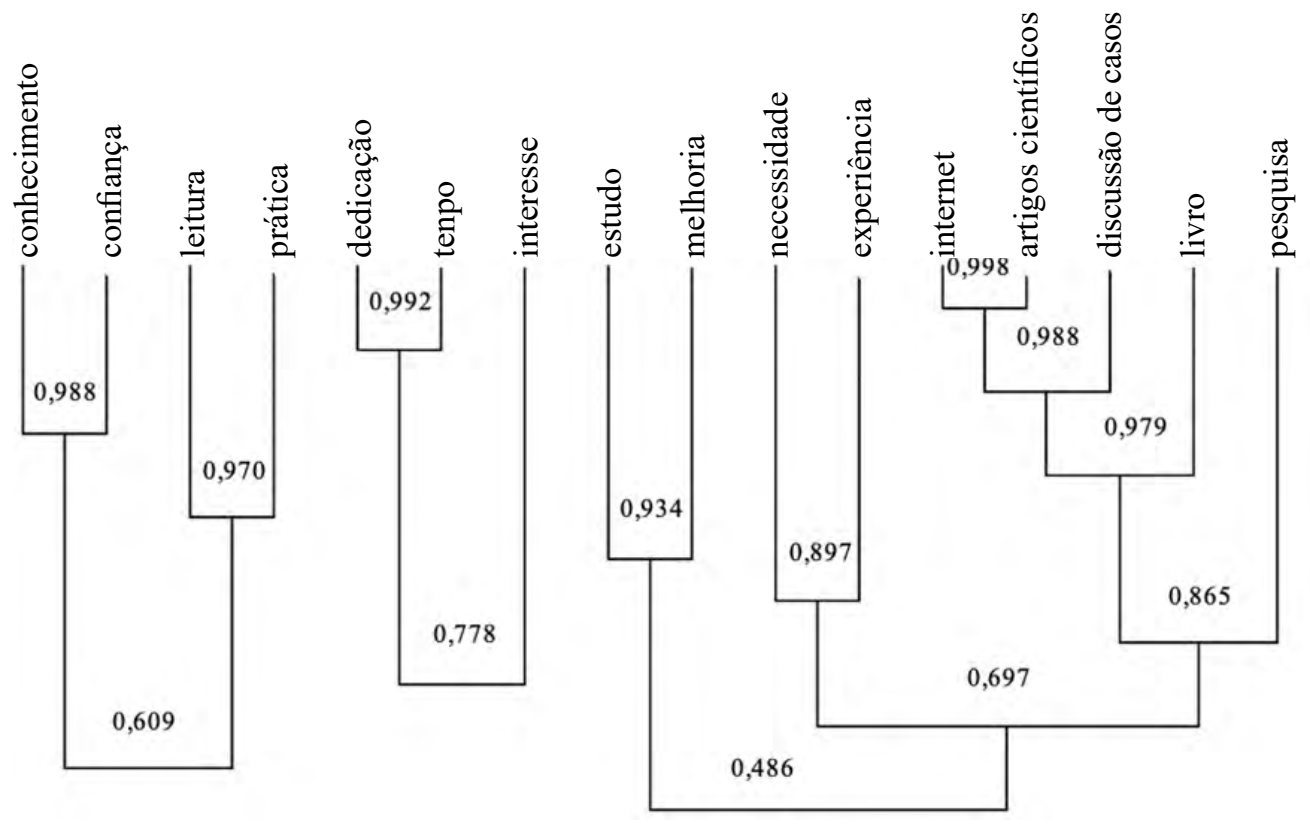


e outro composto por "dedicação", "tempo" e "interesse". E, por último, um grupo maior composto por "estudo", "melhoria", "necessidade", "experiência", "internet", "artigos científicos", "discussão de casos", "livro" e "pesquisa".

A palavra "estudo" aparece no núcleo central e foi a mais evocada (12 vezes) com um rang de 2,083. Nesta pesquisa, o termo "estudo" foi apontado como recurso/ferramenta para atualização e melhora na assistência ao paciente, uma ação proativa e muitas vezes solitária, uma conotação de aquisição de teoria e como sinônimo de pesquisa científica e seu produto:

A gente precisa de estudo pra... pra aprender, então... é ... você precisa mesmo ler, você precisa mesmo... é... sentar pra poder aprender. Eu tive uma formação que foi... não foi assim tradicional. Minha formação foi por PBL. E a gente tinha muito isso... é... o seu estudo é muito sozinho, seu estudo, você não tem muitas pessoas pra poder te ajudar. Você... é... você mesmo vai lá e procura aquilo que você busca. Acho que é isso que... por isso que veio na minha mente nessa hora que o estudo é mais importante (Entrevistado 28).

Porque é o ato em si, você tem que começar, você tem que buscar. O livro tá lá, o conhecimento tá lá, a informação tá lá, você tem que buscar, partir de você. Então acredito que essa é parte mais importante que tem que acontecer a partir de mim (Entrevistado 18).

A palavra "leitura" aparece no núcleo central e foi a segunda mais evocada com conhecimento (dez vezes), com um rang de 2,1. Nesta pesquisa, o termo foi associado a uma ação para quem busca o conhecimento, como o caminho para a teoria.

[...] a gente sempre tem que procurar conhecer mais, mais, através de leitura, desses cursos de ensino a distância, porque sempre tem alguma coisa pra gente aprender (Entrevistado 9).

A leitura propicia a preparação para a execução de ações e atos médicos. Importante inclusive para $o$ aperfeiçoamento e atualização (Entrevistado 47).

A palavra "conhecimento" aparece no núcleo central e foi a segunda mais evocada (dez vezes), com um rang de 2,4 . Nesta pesquisa, o termo foi associado a base, substrato, alicerce para a evolução, sendo algo que é adquirido de forma contínua e como algo infinito, sem se conseguir esgotar. "Conhecimento" é considerado um acúmulo de experiência que pode ser transmitido.

[...] a gente sempre tem que procurar conhecer mais, mais, através de leitura, desses cursos de ensino a distância, porque sempre tem alguma coisa pra gente aprender (Entrevistado 9).

[...] a evolução é o contrário da estagnação. Então quando eu penso em aprendizagem, ... é justamente nessa busca, sempre e contínua pelo saber, né?, pelo conhecimento... então é a maneira da gente evoluir como ser humano, como profissional (Entrevistado 38).

A palavra "dedicação" é a quarta evocação do núcleo central, com um rang de 1,889, evocada nove vezes. Neste estudo, o termo foi associado a uma característica do indivíduo de abdicar de algo para manter o foco, manter-se motivado, tem relação com uma força interior, também a ideia de propósito, pois só se dedica quem se implica e sabe o porquê.

[...] a gente tem que organizar e ter uma gestão, uma organização do estudo com o tempo pra não deixar de fazer outras coisas. De ter uma vida social, descansar, atividade física (Entrevistado 11).

[...] e dedicação... se eu quero me dedicar, eu tenho que ser mais em relação a isso... você tem que tá bem alinhado pra embarcar, porque se não, não dá não. A gente perde... como eu já vi várias vezes, pessoas indo e não conseguindo continuar. Depende muito da gente mesmo. Força de vontade e motivação (Entrevistado 37).

A palavra "necessidade" é a quinta evocação do núcleo central, com um rang de 2,000, evocada sete vezes. Neste estudo, o termo foi associado a um incômodo gerado pelas mudanças, pelo desejo de desempenhar uma boa prática criando uma necessidade.

Acredito que seja necessidade de atualizar, o tempo todo vai mudando a medicina e há essa necessidade (Entrevistado 7).

[...] mesmo que você saiba que aquilo é importante e necessário... não flui, no meu ponto de vista, né?... se você não souber o porquê que você tá fazendo aquilo (Entrevistado 11).

A palavra "prática" é a primeira evocação da primeira periferia, evocada dez vezes, com um rang de 3,700, como estímulo da aprendizagem e para sua fixação, como contraponto à teoria, como uma "validadora" da teoria.

Que a prática que gera a demanda pra eu estudar aquilo. Por exemplo, hoje eu atendi um caso que eu não sabia cem por cento as condutas, né?, melhores a serem tomadas, isso vai me levar a buscar novos conhecimentos. Então, eu vejo a prática como estímulo para buscar novos conhecimentos, fazer novos cursos, buscar novos livros, nova... novos... seguir novos caminhos (Entrevistado 6).

Porque eu sinto que no meu... eu aprendi mais na prática. Praticando a medicina, com essas práticas. Acho que eu aprendi mais com o paciente, na parte, principalmente, da saúde da família, uma parte muito psicológica. Eu aprendi mais a lidar com o paciente, com a medicina (Entrevistado 19). 
A palavra "internet" é a segunda evocação da primeira periferia, evocada oito vezes, com um rang de 2,875. Apesar de evocada, ela não aparece nos discursos de forma explícita, mas foi considerado um lócus democrático, de fácil acesso, com bastante conteúdo e informações: “Porque é onde, eu acho, que tem mais conteúdo pra procurar. $\mathrm{E}$ onde você tem mais informações com acesso" (Entrevistado 23).

A palavra "tempo" é a terceira evocação da primeira periferia, evocada sete vezes, com um rang de 3,0. Nos discursos, o termo traz uma ideia de um recurso finito, necessário de ser administrado, além de indicar a ideia de acúmulo em forma de experiência.

Pela questão de tempo hoje em dia... e prioridades dentro da medicina, né?... a questão assim de trabalho, excesso, [...] quantidade de informação que a gente tem... e dedicação... se eu quero me dedicar, eu tenho que ser mais em relação $a$ isso... você tem que tá bem alinhado pra embarcar, porque se não, não dá não. A gente perde... como eu já vi várias vezes, pessoas indo e não conseguindo continuar. Depende muito da gente mesmo. Força de vontade e motivação (Entrevistado 37).

Porque com o tempo, igual você forma, você sai cru. Você vai buscando conhecimento e melhora. Sempre melhorando, se não fica ultrapassado (Entrevistado 42).

A palavra "experiência" é a primeira evocação da segunda periferia, evocada seis vezes, com um rang de 2,833. Pode-se perceber um sentido de que experiência é resultado de vivência, prática repetida levando a um aprendizado consolidado.

Porque eu acho que através da... das vivências, né?... experiência [...] achoque mais ou menosnesse sentido... que aí você consegue ter todos os seus aprendizados, [...] não adianta nada você quer estudar, por exemplo, uma coisa que você não tá ali, tendo essa vivência, né?... que isso vai acabar se perdendo... isso não fixa na memória (Entrevistado 34).

A palavra "melhoria" é a segunda evocação da segunda periferia, evocada cinco vezes, com um rang de 3,4. O sentido do termo, nos discursos, é de ficar mais completo, capaz de realizar de forma mais plena o trabalho, ter mais conhecimentos e experiências.

É o objetivo [...] a gente quer aprender, mais... e [...] a gente quer especializar e aprender mais, melhorar mais, para, no caso médico, otimizar o cuidado pra pacientee melhorar o trabalho, no meu caso, aqui dentro do posto de saúde, por exemplo (Entrevistado 39).

A expressão "artigos científicos" é a terceira evocação da segunda periferia, evocada quatro vezes, com um rang de 2,750 . Reforça uma ideia de confiabilidade, por se tratar de algo "científico", além de ser mais atual e aplicável a uma situação mais "real", traz uma ideia de evidência, sendo mais uma vez um sentido de algo mais prático e menos teórico.

Porque a medicina baseada em evidência, ela leva em consideração tanto a parte científica, né?, que são os artigos publicados sobre o tema, quanto leva em consideração aquilo que a gente vê no nosso dia a dia. Então, às vezes, eu tenho uma certa opção de um tratamento ou de um... uma... uma ferramenta diagnóstica pra um caso, né?... pra diagnosticar uma doença específica, vamos supor... só que naquele... no meu caso específico, no meu ambiente de trabalho, naquele paciente específico, eu não conseguir utilizar. Então a medicina baseada em evidência, além de trazer o que é de ponta, em termos de inovação e o que é melhor pro paciente, ela adéqua à situação. Então, pra mim, é o mais importante (Entrevistado 30).

A palavra "interesse" é a quarta evocação da segunda periferia, evocada quatro vezes, com um rang de 4,000. O sentido do termo remete à palavra interessante, algo que aguça curiosidade, o desconhecido que deseja se tornar conhecido, oposto a simples; é o complexo e difícil, mas interessante.

\begin{abstract}
Apesar de que tem doença que a gente acaba achando que não é importante, mas são muito importantes, só porque elas têm muita prevalência. [...] a medicina fica assim, né?... alguns colegas, não sei se são todos. Acho que têm muitos que gostam também, né? Acha que, por exemplo, hipertensão não é interessante, mas você vê que muita gente tem até dificuldade de manejar uma hipertensão... (Entrevistado 12).
\end{abstract}

A palavra "confiança" é a quinta evocação da segunda periferia, evocada quatro vezes, com um rang de 4,250. Nos discursos, a palavra "confiança" não aparece, mas alguns discursos em que o termo foi evocado parecem indicar o sentido de sentir-se seguro para tomar uma atitude: "Porque, a partir do conhecimento, eu vou ter segurança para tomar atitudes, fazer o diagnóstico correto, manejar o paciente e me sentir uma profissional mais completa" (Entrevistado 43).

A expressão "discussão de casos" é a sexta evocação da segunda periferia, evocada quatro vezes, com um rang de 4,250. Aponta o sentido de contextualização e forma de estruturação de ideias, além de uma perspectiva de troca de experiências com outra pessoa: "na hora que você tá discutindo um caso [...] parece que você... você grava mais, você... surge mais dúvidas. Na hora que você vai explicar alguma coisa, também alguma dúvida... um colega seu tá discutindo com você" (Entrevistado 25).

O primeiro elemento de contraste é a palavra "pesquisa" evocada cinco vezes, com um rang de 2,200. A palavra traz o sentido da busca do conhecimento, as ações estruturadas para encontrar alguma informação: "Mas comigo é assim, eu acho que só fixa quando você tá ali pesquisando e vivendo aquilo, né?... que aí, de certa forma, se isso te traz alguma dúvida, você 
vai pesquisar, vai querer saber, vai estudar, né?... e a partir daí que isso fixa na sua memória..." (Entrevistado 34).

O segundo elemento de contraste é a palavra "livro" evocada cinco vezes, com um rang de 2,400. O termo traz o sentido de uma fonte de conhecimento, a objetificação do teórico, como um contraste ao que está "fora do livro", que traz a ideia de prático.

O livro tá lá, o conhecimento tá lá, a informação tá lá, você tem que buscar, partir de você... (Entrevistado 18).

[...] na própria consulta tem uma coisa que a gente não vê em livro e a gente às vezes vê numa consulta... (Entrevistado 9).

\section{DISCUSSÃO}

A análise dos resultados permite inferir que o "conhecimento" é o objetivo a ser alcançado pelo médico que dirige sua aprendizagem. Ele traz "confiança" na atuação "prática", e quanto mais ele se torna experiente, mais acumula conhecimento e confiança. Kolb et al. ${ }^{5}$ reforçam esse conceito por meio da explicação do ciclo de aprendizagem, demonstrando que o aprendizado do adulto se baseia em vivência, seguida de reflexão, conceitualização e nova interação. Para esse processo, o sentido que os médicos do estudo trazem é que características individuais de "dedicação", "interesse" e "motivação" são fatores imprescindíveis para a mudança e, no caso, uma "melhoria" da sua atuação. Knowles et al. ${ }^{2}$ constatam que o adulto pode ser influenciado por fatores motivacionais externos, como melhores salários e empregos, porém os fatores motivacionais internos, como satisfação pessoal, realização com o que faz e sentido no que faz, influenciam mais o adulto a aprender, como apontam as análises.

A primeira periferia das representações sociais aqui identificadas traz aspectos processuais da busca por conhecimento. Os entrevistados salientam que as buscas ou "pesquisas" de novas informações, que podem ocorrer pela "internet", por meio da leitura de "livros" e/ou "artigos científicos" ou "discussão de casos", ajudam na consolidação teórica ou no "estudo" e em sua aplicação na "prática". Kolb et al. ${ }^{5}$ apresentam, na Teoria da Aprendizagem Experiencial, a chamada conceitualização, que, depois da vivência e reflexão, o adulto busca consolidar o conhecimento para uma nova aplicação. Esse conceito, presente também na definição da representação social, é reforçado pela primeira periferia, conforme apresentado nos resultados.

A "prática", que aparece na primeira periferia, faz jus ao seu efeito de reforço do núcleo central, pois, no entendimento dos participantes, é quando se desencadeia a percepção por necessidades de conhecimento e ao mesmo tempo que se consolida o conhecimento - ou sua aplicação. Se algum conhecimento é aplicado de forma satisfatória na prática, traz confiança, gera a experiência. Na aprendizagem do adulto, define-se a orientação para aprendizagem voltada a tarefas a serem realizadas ou problemas, que fazem mais sentido para o aprendiz para o enfrentamento de sua realidade, conforme explicitado também nos resultados com relação à atuação na prática em ambientes voltados à resolução de grande parte dos problemas $2,3,32,33$.

Reforça-se ainda, em alguns discursos, que o resultado também da aplicação prática é o aumento da resolutividade e satisfação da pessoa atendida, fator conceitual importante em profissionais atuantes em APS, que lidam com problemas complexos, cuidam ao longo do tempo e, idealmente, apresentam alta resolubilidade ${ }^{32}$. Esse aspecto é explicitado também nos resultados com relação à atuação na prática para resolução de grande parte dos problemas. O cuidado continuado destinado às pessoas na APS faz com que o paciente sempre retorne a esse nível de atenção toda vez que seu problema não foi resolvido ${ }^{2,3,32}$.

Outro elemento da primeira periferia muito importante é o "tempo", apontado tanto como um recurso escasso pelos médicos quanto como fator de mensuração de "experiência" (quem tem mais tempo atuando em algo teria mais experiência). Ele reforça elementos centrais como a "dedicação", no sentido de "gastar" tempo para desenvolver na leitura e estudo, o conhecimento. Além disso, considerando o fato de a autoaprendizagem ser uma necessidade, como apontam os médicos, o tempo precisa ser reservado e priorizado, já que é dado como recurso escasso. Portanto, acaba sendo considerado também uma barreira. A heutagogia também reforça a influência do tempo tanto como limitador quanto como medida daquilo que o aprendiz deseja aprender e, por isso, dedica-se àquilo com mais sentido e buscando direcionar suas próprias necessidades. O tempo como barreira, tal qual ocorre nas representações sociais dos médicos do estudo, também é citado por Knowles como obstáculo ou bloqueio para a motivação ${ }^{2-4}$.

A zona de contraste do quadro de quatro casas é composta por elementos com baixa frequência, mas considerados importantes pelos sujeitos. Assim, reforçam as noções presentes na primeira periferia, podendo apontar o contraste de algum elemento que está sendo perdido por uma representação social ou apontar a existência de um subgrupo minoritário portador de uma representação diferente ${ }^{34}$. No presente estudo, a palavra "pesquisa" como a busca por informações parece reforçar vários aspectos da primeira periferia como a "internet", possível lócus de pesquisa. A palavra "livro", considerando seu espaço nas representações sociais desses sujeitos (zona de contraste), parece demonstrar 
que a aprendizagem utilizando livros, como fonte de consulta, de pesquisa, é um elemento que pode sair dessa representação em breve. Entretanto, pode também ser indicativo de um subgrupo minoritário. A diferença de idade e formação poderia ser um fator relevante para tais possíveis subgrupos. Slotnick ${ }^{7}$ descreveu como modalidade de educação permanente menos estruturada e informal a fonte de estudos via leitura de jornais médicos, revistas e livros. O uso de dispositivos móveis e o acesso digital à informação são uma realidade e têm modificado a relação com livros ${ }^{12-14}$ and preferences for their design and content. Results indicated that for the majority of registrars e-resources were the first preference for obtaining clinical information (77.3\%, fenômeno que é compatível com uma provável mudança da representação social.

Os resultados registram que cerca de um terço dos participantes fazia alguma atividade relacionada à aprendizagem EaD, fato que, associado a um contexto de abundância de informações, pode refletir a "necessidade", como aparece nas entrevistas, de não ficar desatualizado, de acompanhar as evoluções do conhecimento. Deve ser considerado um alerta o fato de que as outras atividades de aprendizagem que os médicos citaram demonstram formatos educacionais com desenho instrucional mais centrado nas necessidades do professor em cumprir objetivos de aprendizagem e menos na autonomia defendida por autores da heutagogia ${ }^{4,35}$.

\section{CONCLUSÕES}

A forma como o médico aprende vem mudando com a inserção de tecnologia tanto para o cuidado quanto para aquisição de conhecimento. A prática baseada em evidências reforça a necessidade de criação de competências relacionadas ao aprender e não somente ao saber, já que o profissional tem de lidar, de forma criativa, com a falta de tempo, a mudança das evidências científicas e o excesso de informações, que podem não ser confiáveis ou exigir interpretação cautelosa.

Ainda que hoje a educação permanente se dê predominantemente por linhas-guia, sumários de evidências e protocolos, "artigos científicos" carregam um simbolismo de poder e evidências científicas que se confundem com a própria busca pelo saber como mecanismo de validação da prática clínica. O saber médico implica aplicar o conhecimento para o cuidado a quem o vem consultar, embasando e empoderando a sua prática dentro de um lócus socialmente construído e culturalmente reconhecido, justamente pelo processo de saber acessar e interpretar essas informações.

Há uma necessidade de ampliar o conhecimento sobre formas mais autônomas de aprendizagem, que não envolvam apenas um ambiente centrado no professor, sendo a EaD um exemplo disso. Tal premissa é especialmente verdadeira na APS, na qual o médico encontra pouca oportunidade de discussão com seus pares de categoria profissional, dado o isolamento de cada unidade, se comparada, por exemplo, com ambulatórios de especialidades ou hospitais. Cabe aqui apontar a limitação que a ausência de trocas entre pares traz, colocando o modelo fundamentado em práticas individuais como uma forma de aprendizagem. Por sua vez, a capacidade trazida pela internet de conexão com outros profissionais e a colaboração também proporcionam diferentes elementos para uma formação mais ampla e satisfatória para essa realidade.

Reforça-se a necessidade de entendimento sobre como esse processo de aprendizagem determinada pelo indivíduo acontece e como a influência dos fatores individuais e sociais e do contexto poderia proporcionar uma melhor formação e inserção em currículos de formação formal como graduação e pós-graduação. Tal processo se dará necessariamente pela tarefa fundamental de ensinar o médico a aprender, ou seja, ensinar a acessar as informações, avaliar sua qualidade, analisar a evidência e aplicá-la à situação em voga, embasando sua prática com a melhor informação disponível, estabelecendo o aprendizado contextual e significativo. Além disso, ele deverá ser capaz de se autoavaliar, aprimorando sua capacidade de aprender de forma contínua e efetiva.

\section{CONTRIBUIÇÃO DOS AUTORES}

Gustavo Valadares Labanca Reis participou da concepção da pesquisa, da coleta e análise dos dados, da elaboração do artigo e da revisão crítica do texto. Cristina Andrade Sampaio e Antônio Prates Caldeira orientaram todas as etapas da pesquisa e do desenvolvimento do manuscrito, e participaram da coleta e análise dos dados, da elaboração do artigo e da revisão crítica do texto. Jucimere Fagundes Durães Rocha e Leonardo Cançado Monteiro Savassi participaram da coleta e análise dos dados, e da revisão crítica do texto.

\section{CONFLITO DE INTERESSES}

Declaramos não haver conflito de interesses.

\section{FINANCIAMENTO}

Declaramos não haver financiamento.

\section{REFERÊNCIAS}

1. Boyd RD. Redefining the discipline of adult education. San Francisco: Jossey-Bass; 1980.

2. Knowles MS, Holton III EF, Swanson RA. Aprendizagem de resultados: uma abordagem prática para aumentar a efetividade da educação corporativa. 2a ed. Rio de Janeiro: Elsevier; 2011.

3. Knowles M. The modern practice of adult education: andragogy versus pedagogy. New York: Associated Press; 1970. 
4. Hase S, Kenyon C. From andragogy to heutagogy. ultiBASE Article. 2001;5:1-10.

5. Kolb AY, Kolb DA. The Kolb Learning Style Inventory 4.0 - a comprehensive guide to the theory, psychometrics, research on validity and educational applications. Experience based learning systems In: Kolb DA. Experiential learning: experience as the source of learning and development. Upper Saddle River, NJ: Pearson FT Press; 2013.

6. Brown JM, Patel M, Howard J, Cherry G, Shaw NJ. Changing clinical practice: significant events that influence trainees' learning. Educ Prim Care. 2011;22(1):25-31 [access in 27 jul 2021]. Available from: http:// pesquisa.bvsalud.org/portal/resource/pt/mdl-21333128.

7. Slotnick HB. How doctors learn: physicians'self-directed learning episodes. Acad Med. 1999 Oct;74(10):1106-17 [access in 27 jul 2021]. Available from: http://www.ncbi.nlm.nih.gov/pubmed/10536633.

8. Davis $D, O$ 'Brien $M$, Thomson MA, Freemantle $N$, Wolf $F$, Mazmanian $P_{1}$ et al. Impact of formal continuing medical education: do conferences, workshops, rounds, and other traditional continuing education activities change physician behavior or health care outcomes? JAMA. 1999;282(9):867-74.

9. Mansouri M, Ocelyn L. A meta-analysis of continuing medical education effectiveness. J Contin Educ Health Prof. 2007;27(1):6-15.

10. Davis D. Does CME work? An analysis of the effect of educational activities on physician performance or health care outcomes. Int J Psychiatry Med. 1998;28(1):21-39.

11. Fox RD, Bennett NL, Rider MS. Continuing medical education: learning and change: Implications for continuing medical education. Int J Technol Assess Heal Care. 1998;316(7129):466-8 [access in 27 jul 2021]. Available from: https://www.ncbi.nlm.nih.gov/pmc/articles/PMC2665605/pdf/9492684.pdf.

12. Denny B, Chester A, Butler M, Brown J. Australian GP registrars' use of e-resources. Educ Prim Care. 2015;26(2):79-86 [access in 27 jul 2021]. Available from: http://pesquisa.bvsalud.org/portal/resource/pt/mdl25898296.

13. Dimond R, Bullock A, Lovatt J, Stacey M. Mobile learning devices in the workplace: "As much a part of the junior doctors" kit as a stethoscope'? BMC Med Educ. 2016;16(1):1-9. doi: 10.1186/s12909-016-0732-z.

14. Lumsden CJ, Byrne-Davis LMT, Mooney JS, Sandars J. Using mobile devices for teaching and learning in clinical medicine. Arch Dis Child Educ Pract Ed. 2015 Oct;100(5):244-51. doi: 10.1136/archdischild-2014-306620.

15. Cisne Tomaz JB, Mamede S, Coelho Filho JM, de Filho JSR, Van Der Molen HT. Effectiveness of an online problem-based learning curriculum for training family medical doctors in Brazil. Educ Heal Chang Learn Pract. 2015;28(3):187-93 [access in 18 abr 2019]. Available from: http://www. educationforhealth.net/temp/EducHealth283187-7938078_220300.pdf.

16. Tomlinson J, Shaw T, Munro A, Johnson R, Madden DL, Phillips R, et al. How does tele-learning compare with other forms of education delivery? A systematic review of tele-learning educational outcomes for health professionals. N S W Public Health Bull. 2013;24(2):70-5.

17. Oliveira V, Savassi L, Lemos A, Campos F. eLearning for health in Brazil UNA-SUS in numbers. J Int Soc Telemed eHealth. 2016;4:e9. [access in 6 jul 2021]. Available from: https://journals.ukzn.ac.za/index.php/JISfTeH/ article/view/139.

18. Demarzo MMP, Almeida RCC, Marins JJN, Trindade TG, Anderson MIP, Stein AT, et al. Diretrizes para o ensino na atenção primária à saúde na graduação em Medicina. Rev Bras Educ Med. 2012;36(1):143-8 [access in $15 \mathrm{abr}$ 2021]. Available from: https://www.scielo.br/j/rbem/a/ QSskKsFFqF5BSXwFf6G5qJB/?lang=pt\&format=pdf.
19. Gomes AP, Costa JRB, Junqueira TS, Arcur MB, Siqueira-Baptista R. Atenção primária à saúde e formação médica: entre episteme e práxis. Rev Bras Educ Med. 2012;36(4):541-9.

20. Vieira SP, Pierantoni CR, Magnago C, Ney MS, Miranda RG. A graduação em medicina no Brasil ante os desafios da formação para a atenção primária à saúde. Saúde Debate. 2018;42(esp 1):189-207.

21. Cavalli LO, Rizzotto MLF. Formação dos médicos que atuam como líderes das equipes de atenção primária em saúde no Paraná. Rev Bras Educ Med. 2018;42(1):31-9 [access in 27 jul 2021]. Available from: http://www.scielo. $\mathrm{br} / \mathrm{scielo}$.php?script=sci_arttext\&pid=S0100-55022018000100031.

22. Antônio J, Neto C. Formação médica generalista: percepção do profissional e do estudante. HU Rev. 2014;40(1-2):15-25.

23. Figueiredo A, Santos A, Reis G, Tavares W, Paula H, Savassi L. Aplicação de metodologias ativas na construção de cursos autoinstrucionais a distância pela UNA-SUS/UFOP em interface com as transformações recentes na formação e no cuidado em saúde. Práticas inovadoras da Rede UNA-SUS tecnologias e estratégias pedagógicas para a promoção da educação permanente em saúde. Porto Alegre: Editora da UFCSPA; 2018. p. 59-78.

24. Savassi LCM, Figueiredo AM, Santos AO, Reis GVL, Tavares W, Paula HC. Experiência da UNA-SUS UFOP na construção reversa de cursos a distância baseadosd em trilhas de aprendizagem de múltiplos desfechos. Experiências exitosas da Rede UNA-SUS: trajetórias de fortalecimento e consolidação da educação permanente em saúde no Brasil. São Luis: Edufma; 2017. p. 214-31.

25. Moscovici S. La psychanalse, sonimageetson public. Paris: Press Universitaires de France; 1961.

26. Abric J-C. L 'approche structurale des représentations sociales: devéloppements récents. In: Anais da V Conferência Internacional sobre Representações Sociais ; 1998. México,1998.

27. Reis AOA, Sarubbi Junior V, Bertolino Neto MM, Rolim Neto ML. Tecnologias computacionais para auxílio em pesquisa qualitativa software EVOC. São Paulo: Schoba; 2013. 106 p.

28. Abric JC. A abordagem estrutural das representações sociais. In: Abric JC. Estudos interdisciplinares de representação social. Goiânia: AB; 2000. p. 27-38.

29. Oliveira DC, Marques SC, Gomes AMT, Teixeira MCTV. Análise das evocações livres: uma técnica de análise estrutural das representações sociais. Representações sociais: uma teoria sem fronteiras. Rio de Janeiro: Museu da República; 2005.

30. Vergès P. Os questionários para análise das representações sociais. In Vergès $P$. Perspectivas teórico-metodológicas em representações sociais. João Pessoa: Editora da Universidade Federal da Paraíba; 2005. p. 201-28.

31. Bardin L. Análise de conteúdo. 3a ed. Lisboa: Edições 70; 2011.

32. Starfield B. Atenção primária: equilibrio entre necessidades de saúde, serviços e tecnologia. Brasília: Unesco; 2002.

33. Savassi LCM, Paula HC, Figueiredo AM, Santos AO, Reis GVL. A construção reversa e as trilhas de aprendizagem de múltiplos desfechos como metodologias ativas e contextuais na educação a distância em saúde. Revista de Saúde Digital e Tecnologias Educacionais. 2019;4(II):1-13.

34. Camargo BV, Barbará A, Bertoldo RB. Concepção pragmática e científica dos adolescentes sobre a Aids. Psicol Estud. 2007;12(2):277-84.

35. Agonács N, Matos JF. Heutagogy and self-determined learning: a review of the published literature on the application and implementation of the theory. Open Learning: The Journal Open, Distance and e-Learning. 2019 Jan 15;34(3):1-18. doi: 10.1080/02680513.2018.1562329. 


\section{ERRATA}

Na página 9, linha 10, onde lê-se:

O uso de dispositivos móveis e o acesso digital à informação são uma realidade e têm modificado a relação com livros12-14and preferences for their design and content. Results indicated that for the majority of registrars e-resources were the first preference for obtaining clinical information (77.3\%, fenômeno que é compatível com uma provável mudança da representação social.

\section{Considera-se:}

O uso de dispositivos móveis e o acesso digital à informação são uma realidade e têm modificado a relação com livros ${ }^{12-14}$, fenômeno que é compatível com uma provável mudança da representação social.

Revista Brasileira de Educação Médica, 45(3): e194, 2021. 